\title{
High-grade serous ovarian carcinoma with mucinous differentiation: report of a rare and unique case suggesting transition from the "SET" feature of high-grade serous carcinoma to the "STEM" feature
}

Yuichiro Hatano ${ }^{1 *}$ (D), Maho Tamada', Nami Asano ${ }^{1}$, Yoh Hayasaki ${ }^{2}$, Hiroyuki Tomita', Ken-ichirou Morishige ${ }^{2}$ and Akira Hara ${ }^{1}$

\begin{abstract}
Background: High-grade serous carcinoma, a representative high-grade ovarian carcinoma, is believed to be closely associated with a TP53 mutation. Recently, this category of ovarian carcinoma has gained increasing attention owing to the recognition of morphological varieties of TP53-mutated high-grade ovarian carcinoma. Herein, we report the case of a patient with high-grade serous carcinoma with mucinous differentiation.

Case presentation: A 59-year-old postmenopausal woman was referred to the gynecologist because of abnormal vaginal bleeding. The radiological assessment revealed an intrapelvic multicystic mass, which was interpreted as an early right ovarian cancer and then removed by radical surgery. Histologically, the cancer cells were found in the bilateral ovaries and para-aortic lymph nodes. The cancer cells showed high-grade nuclear atypia and various morphologies, including the solid, pseudo-endometrioid, transitional cell-like (SET) pattern, and mucin-producing patterns. Benign and/or borderline mucin-producing epithelium, serous tubal intraepithelial carcinoma, and endometriosis-related lesions were not observed. In immunohistochemistry analyses, the cancer cells were diffuse positive for p53; block positive for p16; partial positive for WT1, ER, PgR, CDX2 and PAX8; and negative for p40, p63, GATA3, Napsin A, and vimentin. The Ki-67 labeling index of the cancer cells was 60-80\%. Direct sequencing revealed that the cancer cells contained a missense mutation (c.730G >A) in the TP53 gene.

Conclusion: Mucinous differentiation in high-grade serous carcinoma is a rare and unique ovarian tumor phenotype and it mimics the phenotypes of mucinous or seromucinous carcinoma. To avoid the misdiagnosis, extensive histological and immunohistochemical analyses should be performed when pathologists encounter high-grade mucinproducing ovarian carcinoma. The present case shows that the unusual histological characteristic of high-grade serous carcinoma, the "SET" feature, could be extended to the solid, transitional, endometrioid and mucinous-like (STEM) feature.
\end{abstract}

Keywords: Ovary, High-grade serous carcinoma, SET feature, Mucinous differentiation, TP53

\footnotetext{
* Correspondence: yuha@gifu-u.ac.jp

'Department of Tumor Pathology, Gifu University Graduate School of

Medicine, Gifu 501-1194, Japan

Full list of author information is available at the end of the article
}

(c) The Author(s). 2019 Open Access This article is distributed under the terms of the Creative Commons Attribution 4.0 International License (http://creativecommons.org/licenses/by/4.0/), which permits unrestricted use, distribution, and reproduction in any medium, provided you give appropriate credit to the original author(s) and the source, provide a link to the Creative Commons license, and indicate if changes were made. The Creative Commons Public Domain Dedication waiver (http://creativecommons.org/publicdomain/zero/1.0/) applies to the data made available in this article, unless otherwise stated. 


\section{Background}

Traditionally, classification of ovarian tumor is principally based on morphology $[1,2]$. The current five major ovarian epithelial cancers consist of high-grade and low-grade serous, mucinous, clear cell, endometrioid carcinoma [3, 4]. Each ovarian carcinoma histological type shows a specific cellular phenotype and gene expression profile, which resembles that of the normal corresponding epithelium [5]. For example, serous carcinoma cells partially look like the fallopian tubal epithelial cells. In other words, the evidence of cellular differentiation warrants the validity of morphology-based tumor classification in ovarian cancer.

However, despite the communality of tumor cell differentiation, high-grade serous carcinoma (HGSC) quite differs from low-grade serous carcinoma (LGSC) in regard to clinicopathological features $[6,7]$. HGSC is the most aggressive and common ovarian carcinoma that is believed to be closely associated with a TP53 mutation (type II carcinoma), whereas LGSC, as well as the other major ovarian cancer histological types, is one of the indolent ovarian malignancies that develops from the benign and/or borderline counterparts through multi-step carcinogenesis (type I carcinoma) [8]. To distinguish these two serous carcinomas, p53 immunohistochemistry is a useful tool, which is a surrogate molecular test for TP53 mutation [9]. Indeed, HGSC almost always harbors the TP53 mutation [10], which plays an important role in high-grade ovarian carcinogenesis [11]. Notably, five gynecological pathologists of the United States reassessed the TP53 mutation-lacking HGSC of the TCGA study [10], and then concluded that molecular alteration of the TP53 gene is essential for diagnosis of HGSC [12]. In contrast, LGSC rarely harbors the TP53 mutation [13].

Recently, the concept of TP53 mutation-based high-grade ovarian tumors has garnered increasing attention. Soslow et al. reported that high-grade ovarian carcinoma with unusual morphologies, including the solid, pseudo-endometrioid and transitional cell carcinoma-like (SET) pattern, additionally exhibits the typical molecular aberration of type II carcinoma [14]. This alternative HGSC SET variant indicates that the molecular analysis of ovarian carcinoma is necessary to current tumor classification.

We herein report the case of a patient with a HGSC with mucinous differentiation. The ovarian tumor of the present case is regarded as a rare carcinoma and, thus, could possibly be misdiagnosed as a mucinous or seromucinous carcinoma owing to the morphological finding. Recognition of unique characteristics of this tumor could further expand the concept of ovarian type II carcinoma and prevent the underestimation of its malignant potential.

\section{Case presentation}

\section{Clinical history}

A 59-year-old postmenopausal woman (gravida 2, para

2) was referred to the gynecologist because of abnormal vaginal bleeding. She had a past medical history of hyperthyroidism and was on thyroid hormone replacement therapy at presentation. She denied any familial history of ovarian and/or breast cancer. Blood tests revealed that serum CA125 was slightly high (96.2 U/ $\mathrm{mL})$. Pelvic ultrasonography was notable for a polycystic mass, measuring $117 \times 71 \mathrm{~mm}$, adjacent to the normal-appearing uterus. Abdominopelvic computed tomography showed a polycystic and solid mass, measuring $135 \times 92 \times 100 \mathrm{~mm}$, which was connected to the right ovarian vein. In addition, contrast enhanced-magnetic resonance imaging revealed enhancement in the septal area and heterogeneity of intracystic signal intensity, suggesting ovarian mucinous carcinoma (Fig. 1a). Her disease was diagnosed as early ovarian cancer, FIGO Stage IA (cT1aN0M0); then, she received total hysterectomy with bilateral salpingo-oophorectomy, omentectomy, intra-pelvic and para-aortic lymphadenectomy.

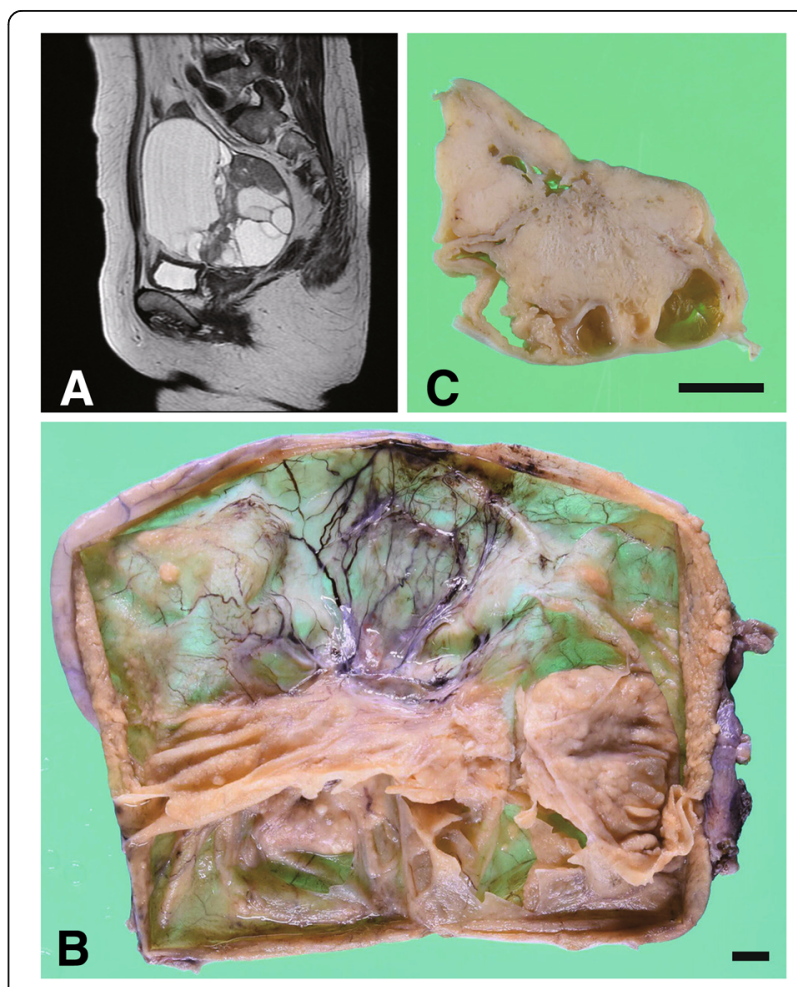

Fig. 1 Overview of the ovarian cancer. a Representative sagittal view of magnetic resonance imaging. The inside (b) and cut surface (c) of the ovarian tumor. Black bars: $1 \mathrm{~cm}$ 


\section{Pathological findings}

The right ovarian tumor contained a serous fluid; the inside was multicystic and partially solid (Fig. 1b and c). Histologically, the cancer cells showed high-grade nuclear atypia and various histological patterns, including solid (Fig. 2a), pseudo-endometrioid (Fig. 2b), and transitional cell-like patterns (Fig. 2c). Such SET-type patterns were observed in approximately $90 \%$ of the tumor, while conventional HGSC histology was limited. In addition, Alcian blue and PAS staining demonstrated that some of the cancer cells contained intracytoplasmic mucin (Fig. 2d-h). The mucinous differentiated foci, which overlapped with other morphological patterns, were approximately $30 \%$ of the tumor, suggesting that the degree of deviation from the mucinous phenotype formed the heterogeneous multicystic image in this tumor (Fig. 1a). The cancer cells had spread into the left ovary and para-aortic lymph nodes, thus confirming the pathological FIGO stage IIIA (pT1bN1aM0).
No benign and/or borderline mucin-producing epithelium, STIC, and endometriosis-related lesion were observed in the extensive histological analysis of the ovarian tumor (total 49 slides) and the fallopian tubes.

In our immunohistochemical analyses, the cancer cells showed diffuse positive staining for p53 (clone: DO-7; Fig. 2i); block positive for p16 (Fig. 2j); partial positive for WT1 (Fig. 2k), ER, PgR (Fig. 2l), CDX2 and PAX8; and negative for p40, p63, GATA3, Napsin A and vimentin (data not shown). The Ki-67 labeling index of the cancer cells was $60-80 \%$ (Fig. $2 \mathrm{~m}$ ).

Since an aberrant p53 expression pattern was displayed by immunohistochemistry, we performed TP53 mutation analysis of ovarian cancer by direct sequencing according to the methods described previously [15]. The results showed that the cancer cells contain a c.730G $>\mathrm{A}$ mutation, which alters glycine to serine in codon 244 of exon 7 in TP53 (Fig. 3). Finally, we diagnosed the
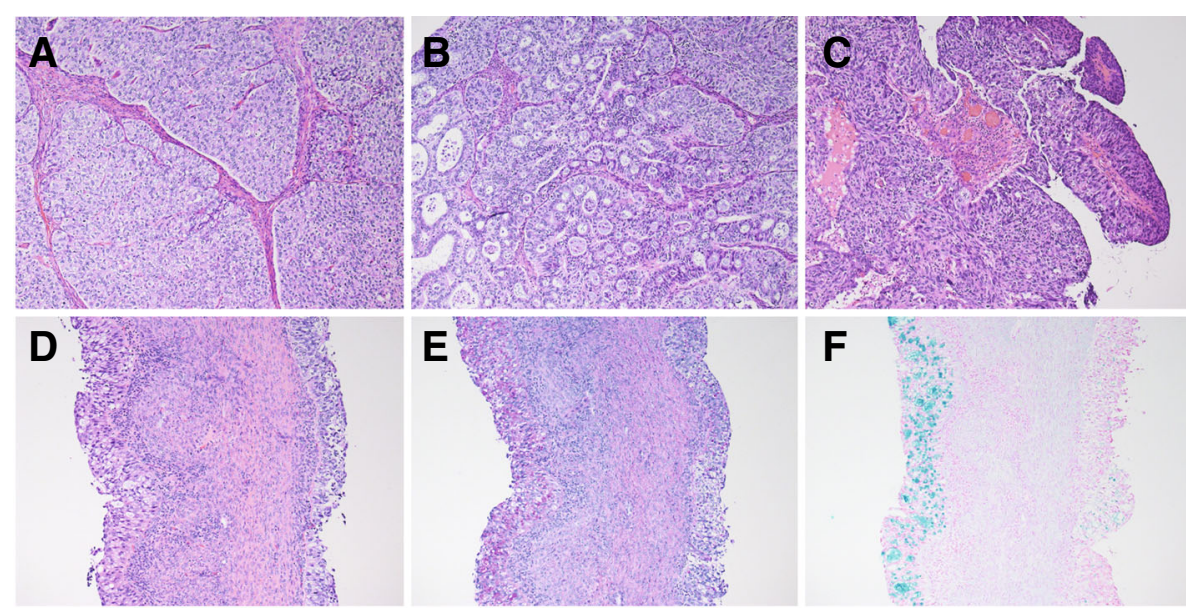

E
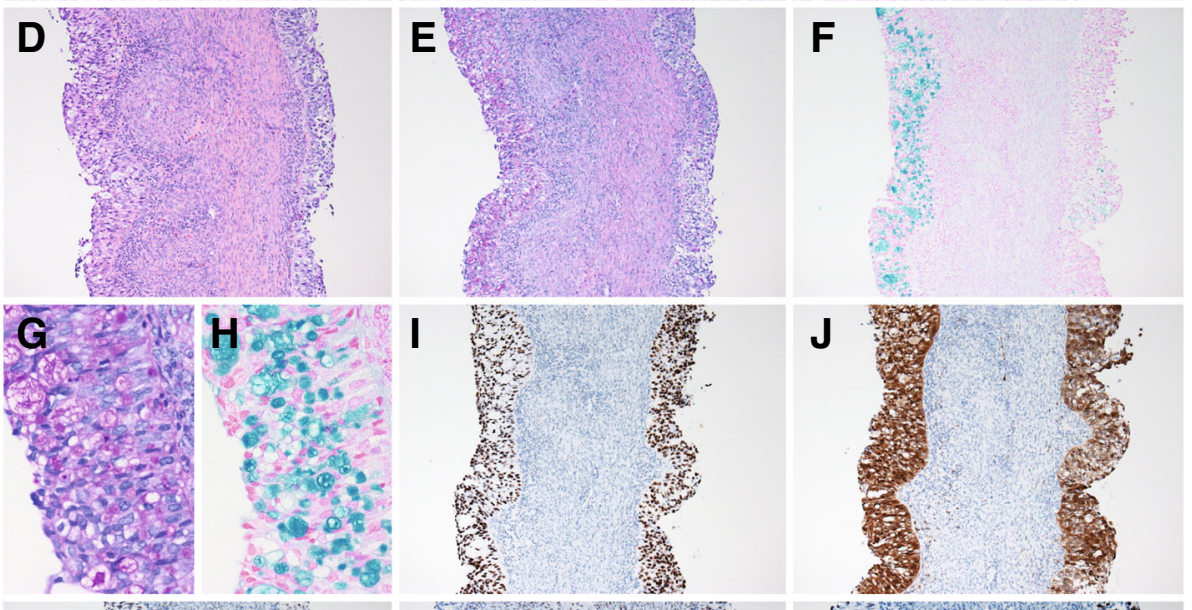

$\mathbf{J}$
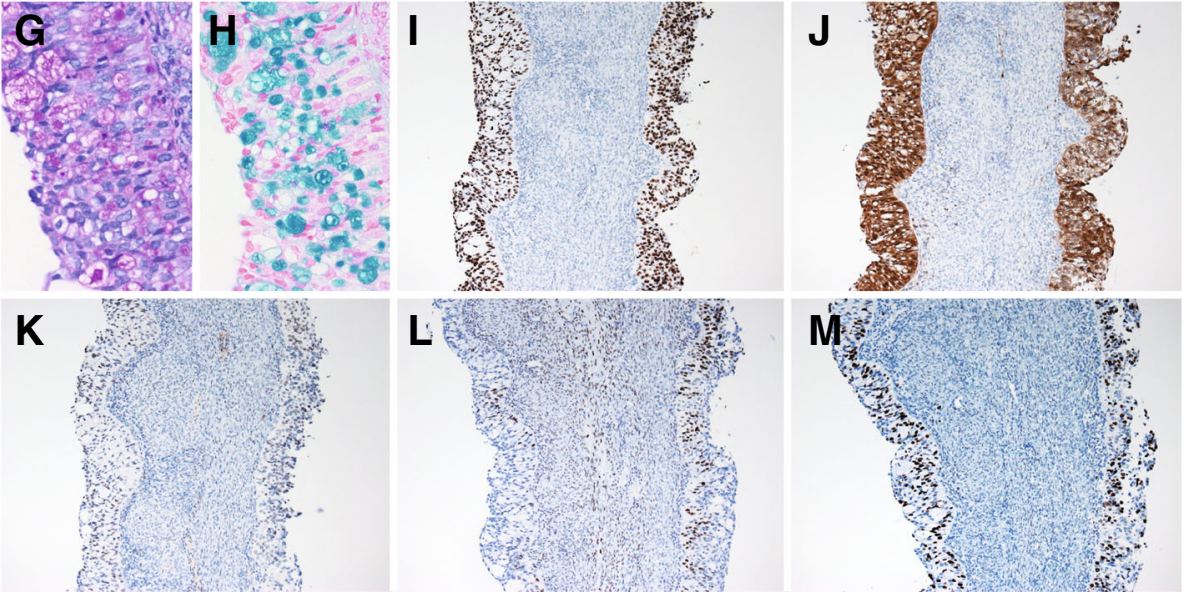

Fig. 2 Pathological findings of the ovarian cancer. a - d the cancer cells show various morphology, including solid (a), pseudo-endometrioid (b) transitional-like (c) and mucin-producing (d) patterns. $\mathbf{e}-\mathbf{h}$ Representative PAS $(\mathbf{e}, \mathbf{g})$ and Alcian blue $(\mathbf{f}, \mathbf{h})$ stained images of mucin-producing cancer cells. Note that ratio of mucin-producing tumor cells is different between the right and left side of septa. $\mathbf{i}-\mathbf{m}$ Representative immunostained images of the p53 (i), p16 (j), WT1 (k), PgR (L) and Ki-67 (m) 
ovarian tumor as a HGSC with SET feature and mucinous differentiation.

\section{Discussion}

In the present case, the cancer cells showed high-grade nuclear atypia and histological heterogeneity, including SET-like and mucin-producing patterns. According to the first and second editions of WHO tumor classification $[1,16]$, mucin production may be found in serous tumors, particularly the serous borderline forms [16], but the mucins are almost entirely extracellular. In contrast, the mucin in the present case seemed to be intracellular and this mucin-producing phenotype mimicked that of a mucinous carcinoma or a minor ovarian epithelial cancer, seromucinous carcinoma [2]. Ovarian mucinous carcinoma contains mucus-scarce cells with marked nuclear pleomorphism [17] and occasionally harbors a TP53 mutation [18, 19], whereas seromucinous carcinoma usually shows endometrioid carcinoma-like morphology and wild-type p53 immunophenotype [20]. In conclusion, these ovarian mucin-producing tumors should be ruled out in the present case.

On the other hand, the high-grade and/or various morphologies of the present case indicated type II carcinoma, such as SET-type HGSC. Consistent with the finding, the immunophenotype of the cancer cells resembled tubo-ovarian transitional cell carcinoma and HGSC [21, 22]. In addition, the relationship between TP53 missense mutation and p53 overexpression is consistent with the recently published article about the correlation between TP53 genotype and p53 immunophenotype in HGSC [23].

As described previously, transitional cell carcinoma is regarded as a variant of HGSC in the current WHO classification [2]. Interestingly, transitional cell carcinoma was defined as malignant transitional cell tumor without benign and/or borderline Brenner component $[16,24]$. However, the majority of this category of ovarian cancers appeared with other histological types of malignant components, including serous, endometrioid, undifferentiated, or unclassified carcinoma [25]. Taking the molecular and morphological findings into consideration, it is reasonable to reclassify this entity of ovarian tumor as a HGSC SET variant. However, SET-type HGSC would be distinct from the conventional-type HGSC due to strong association with $B R C A$ dysfunction that is a potent target of the poly-ADP ribose polymerase (PARP) inhibitor [14, 26-29].

In the present case, the major question was whether transitional cell carcinoma and/or SET-type HGSC show mucinous differentiation. Although the milestone papers of SET-type HGSC lacked the description of whether the SET feature includes the mucinous phenotype $[14,26]$,

\section{T G C A T G G G C G G C 90}
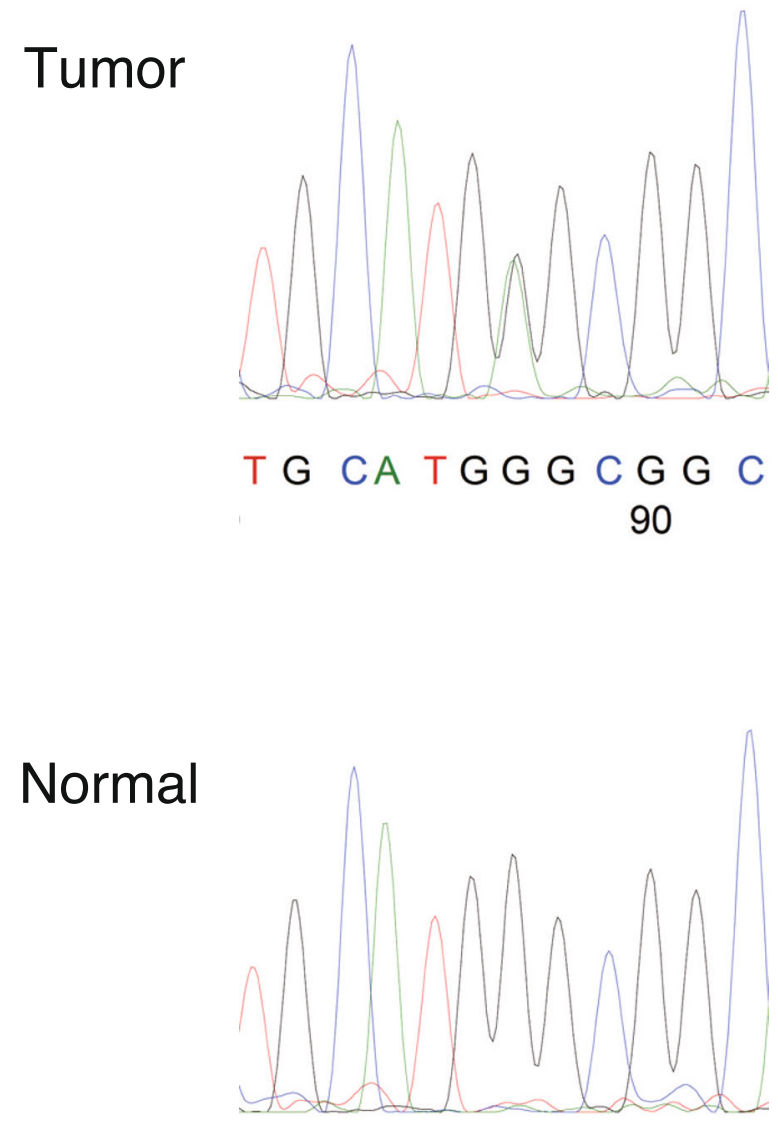

Fig. 3 TP53 mutation analysis of the ovarian tumor. A DNA sequence analysis of TP53 exon 7 from the ovarian tumor and normal tissue with the reverse-primer. The sequence of the ovarian tumor shows biallelic pattern at the coding DNA reference number 730, indicating that HGSC harbors a missense mutation (p.G244S) in the TP53 gene

the AFIP atlas book illustrated the mucin secretion of malignant transitional cell tumor without a Brenner tumor component [30]. In addition, Silva et al. reported that 46 and 9 out of 88 TCC cases contained mucin-producing glands and small microcystic lesion, respectively [25]. Therefore, we believe that this ovarian tumor is a variant of HGSC because of the presence of pure high-grade carcinoma with SET-like morphology and TP53 mutation and the absence of any ovarian benign and/or borderline tumor component, as revealed by the extensive pathological examination.

Besides the morphological pattern and molecular characteristics, the coexisting precursor and/or pre-malignant 
lesion is an important diagnostic clue for ovarian carcinoma. There are two candidate precursors of ovarian mucinous tumor: mature teratoma and Brenner tumor $[31,32]$. In the present case, neither teratoma nor Brenner tumor element was found by radiological and pathological examination. Ovarian endometriosis, which is often associated with an ovarian seromucinous tumor, was also not detected [20]. Incidental lesion of uncertain significance, ovarian epithelial inclusion with mucinous differentiation [33] was also absent. Taken together, the results of our analyses suggested that no suspected premalignant lesions of the both mucinous and seromucinous tumors existed in the present case. In addition, absence of serous tubal intraepithelial carcinoma [34] was consistent with high-grade ovarian carcinoma, especially SET-type HGSC [26].

Surprisingly, mucin-producing phenotypes of HGSC were found in recent studies. Köbel and colleagues tried to establish a sophisticated immunohistochemical panel and algorithm for highly precise and reproducible classification of the five major ovarian carcinomas [3]. Although their attempt seemed to be practically successful, immunohistochemical and genetic findings of minor cases discorded with their morphological diagnosis. It is noteworthy that 2 of the 61 mucinous carcinomas revised the original histological type as HGSC following arbitration using combined biomarker-assisted review and next-generation sequencing. Their crossover of the histological type was similar to that in the present case, although such mucinous carcinoma was estimated to approximately $0.2 \%$ of the total ovarian epithelial carcinomas, according to their data. To clarify the clinicopathological significance of this extremely rare tumor, a case series study would be needed.

\section{Conclusion}

We herein report the case of a HGSC with mucinous differentiation. This rare and unique tumor could be possibly misdiagnosed as a mucinous or seromucinous carcinoma especially when the diagnosis is based solely on morphological assessment of a small amount of histological sections. To avoid the misdiagnosis, extensive histological and immunohistochemical analyses should be performed when pathologists encounter high-grade mucin-producing ovarian carcinoma. In addition to the dedicated workup, we must recall that differential diagnosis of such tumors includes this rare variant of HGSC. In other word, mucinous phenotype in high-grade ovarian cancer cells is a candidate diagnostic clue of the HGSC SET variant. The present case shows that the unusual histological characteristic of high-grade serous carcinoma, the "SET" feature, could be extended to the solid, transitional, endometrioid and mucinous-like (STEM) feature. This comprehensive recognition of high-grade ovarian carcinoma, STEM, would contribute to the establishment of integrated histological and molecular-based tumor classification, which supports precise, reproducible and practical diagnosis and choosing an optimal personalized and/or molecular medicine in the future.

\begin{abstract}
Abbreviation
HGSC: High-grade serous carcinoma; LGSC: Low-grade serous carcinoma; SET: Solid, pseudo-endometrioid and transitional-like; FIGO: The International Federation of Gynecology and Obstetrics; WHO: World Health Organization; PARP: Poly-ADP ribose polymerase; STEM: Solid, transitional, endometrioid and mucinous-like
\end{abstract}

Acknowledgements

Not applicable.

\section{Funding}

This work was supported by JSPS KAKENHI Grant Number JP18K15207.

\section{Availability of data and materials}

The datasets used and/or analyzed during the current study are available from the corresponding author upon reasonable request.

\section{Authors' contributions}

YuH: conception and writing of manuscript. YuH and MT: TP53 mutation analysis. YoH: collection of clinical data. YuH and NA: pathological diagnosis and immunohistochemical analyses. TM, KM and AH: revision of manuscript. All authors read and approved the final manuscript prior to submission.

Ethics approval and consent to participate

Not applicable.

\section{Consent for publication}

Written informed consent was obtained from the patient for the publication of this case report.

\section{Competing interests}

The authors declare that they have no competing interests.

\section{Publisher's Note}

Springer Nature remains neutral with regard to jurisdictional claims in published maps and institutional affiliations.

\section{Author details}

1Department of Tumor Pathology, Gifu University Graduate School of Medicine, Gifu 501-1194, Japan. ${ }^{2}$ Department of Obstetrics and Gynecology, Gifu University Graduate School of Medicine, Gifu 501-1194, Japan.

Received: 9 November 2018 Accepted: 4 January 2019

Published online: 12 January 2019

References

1. Serov SF, Scully RE, Sobin LH. Histological Typing of Ovarian Tumours: International Histological Classification of Tumours No.9. 1st ed. Geneva: WHO; 1973.

2. Kurman RJ, Carcangiu ML, Herrington CS, Young RH. WHO Classification of Tumours of the Female Reproductive Organs. 4th ed. Lyon: WHO Press; 2014.

3. Köbel M, Rahimi K, Rambau PF, Naugler C, Le Page C, Meunier L, et al. An Immunohistochemical Algorithm for Ovarian Carcinoma Typing. Int J Gynecol Pathol. 2016;35:430-41.

4. Prat J, D'Angelo E, Espinosa I. Ovarian carcinomas: at least five different diseases with distinct histological features and molecular genetics. Hum Pathol. 2018;80:11-27.

5. Marquez RT, Baggerly KA, Patterson AP, Liu J, Broaddus R, Frumovitz M, et al. Patterns of gene expression in different histotypes of epithelial ovarian cancer correlate with those in normal fallopian tube, endometrium, and colon. Clin Cancer Res. 2005;11:6116-26. 
6. Malpica A, Deavers MT, Lu K, Bodurka DC, Atkinson EN, Gershenson DM, et al. Grading ovarian serous carcinoma using a two-tier system. Am J Surg Pathol. 2004;28:496-504.

7. Vang R, Shih IM, Kurman RJ. Ovarian low-grade and high-grade serous carcinoma: pathogenesis, clinicopathologic and molecular biologic features, and diagnostic problems. Adv Anat Pathol. 2009;16:267-82.

8. Kurman RJ, Shih IM. The Dualistic Model of Ovarian Carcinogenesis: Revisited, Revised, and Expanded. Am J Pathol. 2016;186:733-47.

9. Altman AD, Nelson GS, Ghatage P, Mclntyre JB, Capper D, Chu P, et al. The diagnostic utility of TP53 and CDKN2A to distinguish ovarian high-grade serous carcinoma from low-grade serous ovarian tumors. Mod Pathol. 2013; 26:1255-63.

10. The Cancer Genome Atlas Research Network. Integrated genomic analyses of ovarian carcinoma. Nature. 2011;474:609-15.

11. Zhang M, Zhuang G, Sun X, Shen Y, Wang W, Li Q, et al. TP53 mutationmediated genomic instability induces the evolution of chemoresistance and recurrence in epithelial ovarian cancer. Diagn Pathol. 2017;12:16.

12. Vang R, Levine DA, Soslow RA, Zaloudek C, Shih IM, Kurman RJ. Molecular Alterations of TP53 are a Defining Feature of Ovarian High-Grade Serous Carcinoma: A Rereview of Cases Lacking TP53 Mutations in The Cancer Genome Atlas Ovarian Study. Int J Gynecol Pathol. 2016:35:48-55.

13. Jones S, Wang TL, Kurman RJ, Nakayama K, Velculescu VE, Vogelstein B, et al. Low-grade serous carcinomas of the ovary contain very few point mutations. J Pathol. 2012;226:413-20.

14. Soslow RA, Han G, Park KJ, Garg K, Olvera N, Spriggs DR, et al. Morphologic patterns associated with BRCA1 and BRCA2 genotype in ovarian carcinoma. Mod Pathol. 2012;25:625-36.

15. Hatano Y, Fukuda S, Makino H, Tomita H, Morishige Kl, Hara A. High-grade serous carcinoma with discordant p53 signature: report of a case with new insight regarding high-grade serous carcinogenesis. Diagn Pathol. 2018;13:24.

16. Scully RE, Sobin LH. Histological Typing of Ovarian Tumours: World Health Organization. In: International Histological Classification of Tumours. 2nd ed. Berlin: Springer-Verlag; 1999.

17. Khunamornpong S, Settakorn J, Sukpan K, Suprasert P, Siriaunkgul S. Primary ovarian mucinous adenocarcinoma of intestinal type: a clinicopathologic study of 46 cases. Int J Gynecol Pathol. 2014;33:176-85.

18. Brown J, Frumovitz M. Mucinous tumors of the ovary: current thoughts on diagnosis and management. Curr Oncol Rep. 2014;16:389.

19. Ryland GL, Hunter SM, Doyle MA, Caramia F, Li J, Rowley SM, et al. Mutational landscape of mucinous ovarian carcinoma and its neoplastic precursors. Genome Med. 2015;7:87.

20. Taylor J, McCluggage WG. Ovarian seromucinous carcinoma: report of a series of a newly categorized and uncommon neoplasm. Am J Surg Pathol. 2015;39:983-92.

21. Magrill J, Karnezis AN, Tessier-Cloutier B, Talhouk A, Kommoss S, Cochrane D, et al. Tubo-Ovarian Transitional Cell Carcinoma and High-Grade Serous Carcinoma Show Subtly Different Immunohistochemistry Profiles. Int J Gynecol Pathol. 2018. https://doi.org/10.1097/PGP.0000000000000538.

22. Takeuchi T, Ohishi $Y$, Imamura H, Aman M, Shida K, Kobayashi H, et al. Ovarian transitional cell carcinoma represents a poorly differentiated form of high-grade serous or endometrioid adenocarcinoma. Am J Surg Pathol. 2013;37:1091-9.

23. Köbel M, Piskorz AM, Lee S, Lui S, LePage C, Marass F, et al. Optimized p53 immunohistochemistry is an accurate predictor of TP53 mutation in ovarian carcinoma. J Pathol Clin Res. 2016:2:247-58.

24. Tavassoli FA, Deville P. World Health Organization Classification of Tumours. In: Pathology and Genetics of Tumours of the Breast and Female Genital Tract. 3rd ed. Lyon: IARC Press; 2003.

25. Silva EG, Robey-Cafferty SS, Smith TL, Gershenson DM. Ovarian carcinomas with transitional cell carcinoma pattern. Am J Clin Pathol. 1990;93:457-65.

26. Howitt BE, Hanamornroongruang S, Lin DI, Conner JE, Schulte S, Horowitz $\mathrm{N}$, et al. Evidence for a dualistic model of high-grade serous carcinoma: BRCA mutation status, histology, and tubal intraepithelial carcinoma. Am J Surg Pathol. 2015;39:287-93.

27. Ledermann J, Harter P, Gourley C, Friedlander M, Vergote I, Rustin G, et al. Olaparib maintenance therapy in platinum-sensitive relapsed ovarian cancer. N Engl J Med. 2012;366:1382-92.

28. Mirza MR, Monk BJ, Herrstedt J, Oza AM, Mahner S, Redondo A, et al. Niraparib Maintenance Therapy in Platinum-Sensitive, Recurrent Ovarian Cancer. N Engl J Med. 2016;375:2154-64.
29. Coleman RL, Oza AM, Lorusso D, Aghajanian C, Oaknin A, Dean A, et al. Rucaparib maintenance treatment for recurrent ovarian carcinoma after response to platinum therapy (ARIEL3): a randomised, double-blind, placebo-controlled, phase 3 trial. Lancet. 2017;390:1949-61.

30. Scully RE, Young RH, Clements PB. Tumors of the Ovary, Maldeveloped Gonads, Fallopian Tube, and Broad Ligament. In: Atlas of Tumor Pathology. 3rd series, Fascicle 23. Washington, D.C.: Armed Forces Institute of Pathology; 1998.

31. Wang Y, Wu RC, Shwartz LE, Haley L, Lin MT, Shih IM, et al. Clonality analysis of combined Brenner and mucinous tumours of the ovary reveals their monoclonal origin. J Pathol. 2015;237:146-51.

32. Wang Y, Schwartz LE, Anderson D, Lin MT, Haley L, Wu RC, et al. Molecular analysis of ovarian mucinous carcinoma reveals different cell of origins. Oncotarget. 2015:6:22949-58

33. Seidman JD, Krishnan J. Ovarian Epithelial Inclusions With Mucinous Differentiation: A Clinicopathologic Study of 42 Cases. Int J Gynecol Pathol. 2017;36:372-6.

34. Lee Y, Miron A, Drapkin R, Nucci MR, Medeiros F, Saleemuddin A, et al. A candidate precursor to serous carcinoma that originates in the distal fallopian tube. J Pathol. 2007;211:26-35.

\section{Ready to submit your research? Choose BMC and benefit from:}

- fast, convenient online submission

- thorough peer review by experienced researchers in your field

- rapid publication on acceptance

- support for research data, including large and complex data types

- gold Open Access which fosters wider collaboration and increased citations

- maximum visibility for your research: over $100 \mathrm{M}$ website views per year

At $\mathrm{BMC}$, research is always in progress.

Learn more biomedcentral.com/submissions 